\title{
Recent advances of uncertainty management in knowledge modelling and decision making
}

\author{
Van-Nam Huynh ${ }^{1}$
}

Published online: 22 August 2017

(C) Springer Science+Business Media, LLC 2017

This special issue of the Annals of Operations Research is dedicated to the fourth edition of The International Symposium on Integrated Uncertainty in Knowledge Modelling and Decision Making (IUKM 2015), which was successfully held in Nha Trang, Vietnam, on 15-17 October, 2015. The IUKM Symposium aims to provide an international forum for exchanges of research results, ideas, and experiences of using applications among researchers and practitioners involved with all aspects of uncertainty management. This conference series started in 2010 at Japan Advanced Institute of Science and Technology, Ishikawa, Japan, under the title International Symposium on Integrated Uncertainty Management and Applications (IUM 2010). The special volume of the Annals of Operations Research 195 (2012) (Huet 2012 ) is associated with this symposium.

IUKM 2015 was jointly organized by The VNU-Hanoi University of Engineering and Technology (VNU-UET), Pacific Ocean University (POU), Hanoi National University of Education (HNUE), Japan Advanced Institute of Science and Technology (JAIST), and the Belief Functions and Applications Society (BFAS). Its proceedings were published by Springer Lecture Notes in Computer Science series (Huet 2015).

The call for this special issue followed IUKM 2015 and was also open to relevant contributions that were not presented at the symposium. In response, 18 papers were submitted, of which about half were extended version of papers presented at IUKM 2015. All submitted papers went through the regular peer-review process of the Annals of Operations Research. As a result of several selection stages, 11 papers were eventually accepted for publication. They are briefly summarized as follows.

In view of the continuing research reported in the literature on nonparametric estimation in diffusion models of financial economics from discrete observed data, the paper by Christian Gourieroux, Hung T. Nguyen, and Songsak Sriboonchitta, entitled "Nonparametric Estimation of a Scalar Diffusion Model from Discrete Time Data: A Survey," provides a return to sources in a simple setting of scalar diffusion models, as well as an overview

Van-Nam Huynh huynh@jaist.ac.jp

1 School of Knowledge Science, Japan Advanced Institute of Science and Technology, 1-1 Asahidai, Nomi, Ishikawa 923-1292, Japan 
of its state-of-the-art, together with an elaboration on the use of local times in statistics of stochastic processes. The research results in the scalar case should shed light on the multivariate case. The main contribution of the paper is: In presenting the main principles of estimation for both stationary and non-stationary cases, it is shown that it is possible to estimate non-parametrically the drift and volatility functions without distinguishing these two frameworks.

The paper by Hien Duy Tran, Uyen Hoang Pham, Sel Ly, and T. Vo-Duy, "Extraction Dependence Structure of Distorted Copulas via a Measure of Dependence," proposes two methods to estimate the measure of dependence $\lambda(C)$ for a continuous bivariate random vector by using conditional copula-based Monte Carlo simulation. Thereafter, this measure is applied to investigate how dependence structure of Gaussian and product copulas are changed after being transformed by proportion hazard $(\mathrm{PH})$ and by Wang's distortion functions. The results show that the $\mathrm{PH}$-transform $g_{P H}$ of extreme value copulas preserves the same structure of dependence while the Wang's $g_{\gamma}$ distorted-normal and -product copulas become more comonotonic when increasing the parameter $\gamma$. It is also established that the Gini's index can be expressed in terms of Sobolev's norm for copulas. Due to benefits of this norm, it could be used to construct a new measure of dependence for multivariate random vectors with dimension $d \geq 3$. Additionally, one could study dependence structures of other distorted families of copula with more general class of distortion functions.

In "Confidence Intervals for the Difference between Normal Means with Known Coefficients of Variation" Suparat Niwitpong and Sa-aat Niwitpong introduce new confidence intervals for the difference between normal means with known coefficients of variation of experiments and provide analytical expressions for the coverage probability and the expected length of each confidence interval. The performances of the proposed confidence intervals in terms of their coverage probabilities and their expected lengths are evaluated and compared using Monte Carlo simulation. It would be interesting for further research to consider the confidence interval between normal means with unknown coefficients of variation.

In the paper entitled "On the Disintegration Property of Coherent Upper Conditional Prevision Defined by the Choquet Integral with Respect to its Associated Hausdorff Outer Measure," by Serena Doria, two important properties are investigated for coherent upper conditional previsions with respect to arbitrary partitions. For linear conditional previsions, defined on a linear space of random variables, the concepts of conglomerability and disintegrability coincide. In an imprecise probability framework, conglomerability with respect to every partition, also called full conglomerability, is required as a rationality axiom for a coherent upper prevision since it assures that it can be coherently extended to coherent conditional previsions for any partition $B$ of $\Omega$. If a coherent upper probability is such that all the sets in the partition have zero probability, then its minimal coherent conditional prevision extension is the vacuous coherent conditional prevision. So to verify the existence of separately coherent conditional previsions coherent with the coherent upper prevision is equivalent to verifying the conglomerability for all countable partitions. A consequence is that a fully conglomerable coherent conditional previsions may fail the disintegration property on a null partition. In this work a coherent upper conditional prevision, defined as the Choquet integral with respect to its associated Hausdorff outer measure, is proven to satisfy the disintegration property and the conglomerative principle on every non-null partition of a metric space with positive and finite Hausdorff outer measure in its Hausdorff dimension.

The paper by Yasuo Sasaki, "Generalized Nash Equilibrium with Stable Belief Hierarchies in Static Games with Unawareness," deals with a recently developed game theoretical framework in which agents may be unaware of some game components. It points out a conceptual problem of an existing equilibrium concept of games with unawareness called generalized 
Nash equilibrium. That is, some equilibria can bring about cognitive dissonances to some agents and thus cannot be understood as a stable convention in the game, in contrast to Nash equilibrium in standard games. To avoid this problem, this paper proposes and characterizes generalized Nash equilibrium with stable belief hierarchies, which can be motivated as a stable convention in a game with unawareness. The analysis is limited to static games, and the extension to other games such as dynamic games would be an interesting topic for further research.

In "Global Optimality Test for Maximin Solution of Bilevel Linear Programming with Ambiguous Lower-Level Objective Function," Puchit Sariddichainunta and Masahiro Inuiguchi address a bilevel linear programming problem with ambiguous lower-level objective function (BLPwFAO). This is a sequential decision making problem despite the follower's imprecisely known criterion causing uncertainty of the follower's rational reactions. In this problem, both of the leader and the follower decision makers share the same linear constraints such as in the network flow problem. The paper assumes that the ambiguous follower's objective function is defined by coefficient vectors located in a convex polytope. Moreover, it applies the maximin solution approach and formulates BLPwFAO as a special kind of three-level programming problem. To expedite the solution procedure, the authors propose a global optimality test based on an inner approximation method and extensively utilize the history of rationality tests to omit the verification process of rationality testing in the follower's problem. Finally, the computational efficiency of the proposed method is compared with other test methods based on vertex enumeration.

The paper by Wen-Tao Guo, Van-Nam Huynh, and Songsak Sriboonchitta, "A Proportional Linguistic Distribution Based Model for Multiple Attribute Decision Making Under Linguistic Uncertainty," addresses the problem of multiple attribute decision making (MADM) with uncertain linguistic information by introducing a proportional fuzzy linguistic distribution model based on the symbolic linguistic model combined with distributed assessments. In this model the evaluation on attributes of alternatives is represented by distributions on the linguistic term set used as an instrument for assessment, and conventional aggregation operators are appropriately extended for attribute aggregation. It is shown that the proposed model could effectively overcome the limitations imposed on previously developed linguistic computational models, while maintaining their advantages. In addition, the applicability of the proposed model as well as it advantages over the so-called evidential reasoning approach for MADM are illustrated by an application in product evaluation.

The paper "A Stochastic Dominance Based Approach to Consumer-oriented Kansei Evaluation with Multiple Priorities," by Hong-Bin Yan, Tieju Ma, Songsak Sriboonchitta, and Van-Nam Huynh, proposes a stochastic dominance based approach to consumer-oriented Kansei evaluation with multiple priorities, which aims to provide decision support to consumers when selecting aesthetic products based on their Kansei preferences, and thus is helpful for marketing or recommendation purposes. On one hand, the concept of stochastic dominance is utilized to build an evaluation function, which derives a fuzzy preference matrix of all the products for each selected Kansei attribute. On the other hand, the importance weights of Kansei attributes, captured by a prioritization of Kansei attributes, together with the concept of fuzzy majority, is incorporated into an aggregation function to obtain the global dominance degrees for the products. An application to the hand-painted Kutani cups in Ishikawa, Japan, was conducted to illustrate the effectiveness and efficiency of the proposed approach. It is shown that the proposed approach can reduce the cognitive burden of quantifying Kansei preferences, making it easy to use in practice; additionally, it incorporates both the fuzzy majority and priority information of Kansei attributes to help consumers find 
their preferred products. A potential extension is to explore the use of the proposed approach to critique-based recommender systems.

In "Neighbor Selection for User-based Collaborative Filtering using Covering-based Rough Sets," Zhipeng Zhang, Yasuo Kudo, and Tetsuya Murai first apply covering-based rough sets to the traditional user-based collaborative filtering (UBCF), and then propose a new covering-based collaborative filtering $(\mathrm{CBCF})$ approach for an active user's neighbor selection. Particularly, CBCF inserts a user reduction procedure into the traditional UBCF, and uses covering reduction to remove redundant users from candidate neighbors of an active user. Experimental results show that, for the sparse data sets that often occur in real recommender systems, $\mathrm{CBCF}$ can provide recommendations with better accuracy and coverage than UBCF. However, CBCF needs the item-attribute matrix information that may not be always available in some datasets. It would be interesting to improve CBCF for a new user's neighbor selection, because unlike an active user, a new user often has insufficient rating information.

In "On the Median in Imprecise Ordinal Problems," Sebastien Destercke studies how sets of Median values can be justified and retrieved as legitimate predictions when dealing with ordinal variables and sets of probabilities. He gives two main justifications: one extends the well-known relation between $L_{1}$ loss and precise probabilities, while the other considers the purely ordinal notion of signed-preferences. He shows that the former path also applies to linear (a.k.a. pinball) losses and to sets of generic quantiles. The results can therefore be applied to various settings where preserving the ordinal nature of data is important, such as ordinal regression, sorting problems in multi-criteria decision making, ranking issues, etc. It would clearly be interesting to explore whether some of the presented results can be extended to other kinds of losses, and to link the current research with more complex prediction problems such as graded multi-label, learning to rank, etc.

The final paper, by Zengchang Qin, Tao Wan, and Hanqing Zhao, "Hybrid Clustering of Data and Vague Concepts Based on Labels Semantics," proposes a new approach of clustering numerical data and vague concepts represented by fuzzy labels. Some new distance measures between data and vague concepts are proposed based on label semantics. This could be useful in handling real-world concept clustering. It extends the applicability of classical clustering models from numerical data to imprecise concepts as demonstrated by an image clustering algorithm.

As a guest editor of this special issue, I would like to express my gratitude to all the authors of submitted papers for their efforts and contributions, and to the numerous reviewers for their expertise and all the hard work allocated to the reviewing task to help maintain the quality standards set by the journal. Finally, I am grateful to the Editor-in-Chief, Professor Endre Boros, for offering me the opportunity to edit this special issue, and the Managing Editor of the journal, Ms. Katie D’Agosta, for her continuous support and help with this publication project.

\section{References}

Huynh, V.-N., Lawry, J., Nakamori Y., (Eds.), (2012). Integrated uncertainty management for decision making. Annals of Operations Research, 195.

Huynh, V.-N., Inuiguchi, M., Denœux T., (Eds.), (2015). In Integrated uncertainty in knowledge modelling and decision making - 4th international symposium (IUKM 2015), proceedings. Lecture notes in computer science (Vol. 9376). Springer-Verlag. 